\title{
Erratum to: Pharmacokinetic-pharmacodynamic relationship of bosutinib in patients with chronic-phase chronic myeloid leukemia
}

\author{
Poe-Hirr Hsyu • Diane R. Mould • Richard N. Upton • \\ Michael Amantea
}

Published online: 27 February 2013

(c) Springer-Verlag Berlin Heidelberg 2013

\section{Erratum to: Cancer Chemother Pharmacol (2013) 71:209-218 \\ DOI 10.1007/s00280-012-1998-4}

The author would like to correct the errors in the publication of the original article. The corrected details are given below for your reading.

The first paragraph under heading "Safety Parameters" should read as

"Data from the pooled safety analysis (Appendix Table A2) demonstrated that although gastrointestinal toxicities (e.g., diarrhea [77\%], nausea [40\%], and vomiting [35\%]) were frequently reported across the two studies, most events were of low severity grade. Other toxicities of clinical interest included thrombocytopenia (29\%), rash (31\%), ALT elevation (21\%), AST elevation (17\%), and neutropenia $(16 \%)$."

Secondly, the last sentence in the page 216 beneath the heading "Discussion" should read as

"These data compare favorably with the findings from previous studies of patients with CP CML treated with dasatinib or nilotinib in the second- or third-line settings."

The online version of the original article can be found under doi:10.1007/s00280-012-1998-4.

P.-H. Hsyu $(\bowtie) \cdot$ M. Amantea

Pfizer Inc, 10646 Science Center Drive, La Jolla, CA, USA

e-mail: Poe-Hirr.Hsyu@pfizer.com

D. R. Mould · R. N. Upton

Projections Research Inc, Phoenixville, PA, USA
Finally, Fig. $2 \mathrm{~g}$ has a wrong indication inside the figure label. It should correctly read as

"E $E_{\max } "$ model

The revised figure is placed in the following page. 

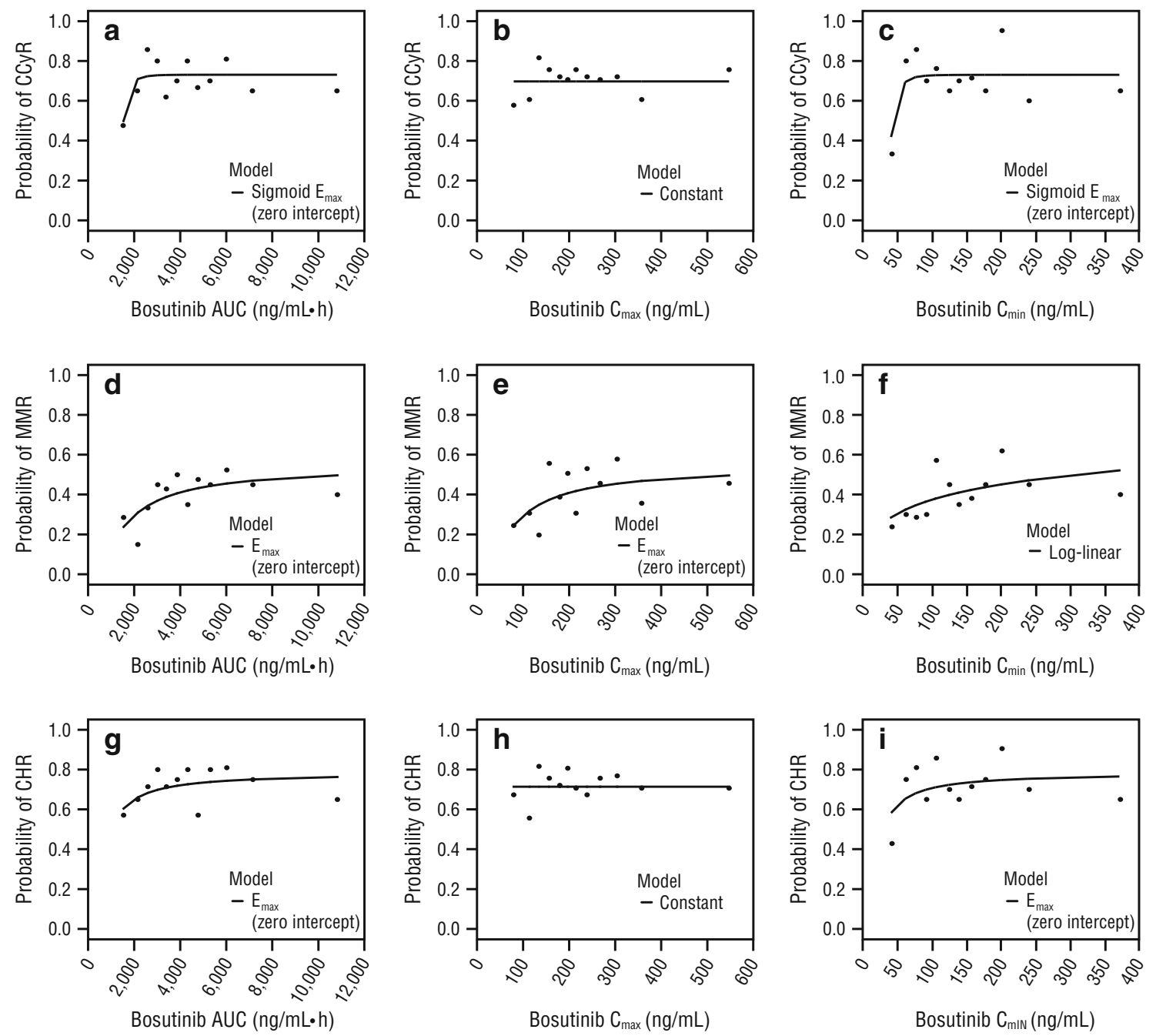

Fig. 2 Probability of response at 1 year versus bosutinib exposure in patients with newly diagnosed CP CML. a, b, and $\mathbf{c}$ are the probability of achieving a CCyR at 1 year versus $\mathrm{AUC}, C_{\max }$, and $C_{\min }$, respectively. $\mathbf{d}, \mathbf{e}$, and $\mathbf{f}$ are the probability of achieving a MMR versus AUC, $C_{\max }$, and $C_{\min }$, respectively. $\mathbf{g}, \mathbf{h}$, and $\mathbf{i}$ are the probability of achieving/maintaining a CHR versus AUC, $C_{\max }$, and $C_{\text {min }}$, respectively. Response data were coded as binary variables, where 0 corresponded to no response and 1 corresponded to response;

exposure metrics were derived from a previously developed bosutinib population pharmacokinetic model. Solid lines are predictions of the best models. Solid lines are predictions of the best models. $C P C M L$ chronic-phase chronic myeloid leukemia, $C C y R$ complete cytogenetic response, $A U C$ area under the curve, $C_{\max }$ maximal plasma concentration, $C_{\min }$ minimum plasma concentration, $M M R$ major molecular response, $\mathrm{CHR}$ complete hematologic response 